\title{
On the Same Wavelength: Face-to-Face Communication Increases Interpersonal Neural Synchronization
}

\author{
Kyongsik Yun \\ Computation and Neural Systems, Division of Biology, California Institute of Technology, Pasadena, California 91125 \\ Review of Jiang et al.
}

Understanding neural mechanisms of social interaction is important for understanding human social nature and for developing treatments for social deficits related to disorders such as autism. However, conventional cognitive and behavioral neuroscience has concentrated on developing novel experimental paradigms and investigating human-computer interactions, rather than studying interpersonal interaction per se. To fully understand neural mechanisms of human interpersonal interaction, we will likely have to investigate human behavior and neural processes in face-to-face social interaction rather than human-computer interaction. Recently, simultaneous EEG or functional near-infrared spectroscopy (fNIRS) has been used to record brain activity of two participants in a face-to-face setting (i.e., hyperscanning) to investigate human social interaction in a more naturalistic context (Jiang et al., 2012; Yun et al., 2012).

In a recent article published in The Journal of Neuroscience, Jiang et al. (2012) investigated interbrain neural synchronization during face-to-face communication using fNIRS hyperscanning. fNIRS measures changes in the regional cerebral blood flow

Received Jan. 7, 2013; revised Jan. 31, 2013; accepted Feb. 4, 2013.

Many thanks to Shinsuke Shimojo for helpful comments on this manuscript.

Correspondence should be addressed to Kyongsik Yun, Computation and Neural Systems, Division of Biology, California Institute of Technology 139-74, 1200 E. California Blvd., Pasadena, CA 91125. E-mail: yunks@caltech.edu.

DOI:10.1523/JNEUROSCI.0063-13.2013

Copyright $\odot 2013$ the authors $\quad 0270-6474 / 13 / 335081-02 \$ 15.00 / 0$ by quantifying the changes in oxyhemoglobin concentration. Given that the left hemisphere is dominant for language function and that the left inferior frontal cortex and inferior parietal cortex have been known to be closely related to action understanding and imitation, i.e., mirror neuron system, fNIRS was placed only on the left hemisphere. The inferior frontal cortex has been particularly associated with empathy and social cognition (Farrow et al., 2001). Jiang and colleagues found a significant increase in cross-correlation between changes in activity of two participants' left inferior frontal cortices only during a dialog in which subjects faced each other (face-to-face), but none during other communication conditions, including dialog in which subjects faced away from each other (back-to-back), monologue with subjects facing each other, and monologue with subjects facing away from each other. Moreover, quality of communication in each condition was assessed by self-report, and face-to-face dialog represented a higher quality of communication than back-to-back dialog. These results suggest that face-to-face conversation has special features that other types of communication lack and that interbrain correlated activity may be an underlying neural process of successful face-to-face communication. In this Journal Club article, I consider four important points regarding the study: (1) validity of the interbrain neural synchrony; (2) limitations that arise from using verbal communication; (3) aptness of fNIRS methodology to face-to-face com- munication; and (4) analysis of spatiotemporal functional connectivity.

Validation of synchronization was strict and appropriate in this study. Jiang et al. (2012) verified that the increase in neural synchronization was specific for pairs of participants using a cross-validation entailing shuffling between participants and calculating interbrain correlations. They found no significant increase in synchronization between randomly paired participants in any of the communication conditions. The results indicate that the interbrain correlated activity is pair-specific and the increased correlation was not by chance or artifacts. Moreover, the time-series of the coherence values were randomly separated into two parts and the averaged coherence values were compared between two parts. No significant difference was found, indicating that the coherence values are consistent across time. Last, Jiang et al. (2012) used other mirror neuron regions, including the premotor area and inferior parietal cortices, to test whether the neural synchronization is region-specific. They found no significant difference in any of the conditions. The results suggest that neural synchronization is specific to the inferior frontal cortex and that this synchrony was primarily contributed by face-to-face social interaction rather than mere mirroring the action of speaking. The pair and region specificity of the results and the temporal consistency of the coherence values successfully validate the robustness of the data.

It is important to note that face-to-face dialog represented a higher quality of 
communication than back-to-back dialog (Arnal et al., 2009). Therefore, the stronger interbrain correlation in face-to-face dialog could result from either a higher quality of communication or from the face-to-face setting, in which various nonverbal cues were present. To deal with this issue, Jiang et al. (2012) performed an additional analysis wherein they separated time points showing the nonverbal communication between participants, such as turn-taking behavior and body language. Time points in which facial expression and gestures occurred showed significant neural synchronization compared with other time points in the face-to-face dia$\log$ condition only. The results suggest that the increased interbrain correlation mainly reflected nonverbal interaction. However, it is still possible that the quality of communication influences interbrain neural synchronization. A previous study suggests that changes in speech amplitude can be synchronized with the brain activity of a listener (Chandrasekaran et al., 2009). Successful temporal synchrony between two participants' dialog and brain activity increased the signal-to-noise ratio of neural signals and thus helped to improve quality of communication (Ahissar et al., 2001; Luo and Poeppel, 2007).

A major advantage of fNIRS is that the instrument is more portable and inexpensive for functional neuroimaging than functional magnetic resonance imaging ( $\mathrm{fMRI}$ ). In addition, fNIRS is robust to movement artifacts compared with EEG and fMRI, allowing investigation of language processes, infants, and various neuropsychiatric patients who cannot remain sufficiently still for fMRI (Bunce et al., 2006). fNIRS is especially appropriate for a face-to-face dialog experimental setting in that fMRI does not allow us the study of a face-to-face condition and EEG is vulnerable to movement artifacts, including those produced by vocalization, facial expression, and gestures involved in dialog. Moreover, fMRI gradient noise often exceeds $100 \mathrm{~dB}$ and thus interferes with auditory stimulation, which is crucial for communication experiments (Plichta et al., 2011). Some argue that fNIRS may not be appropriate for studies of the adult human brain, because the light path is grossly affected by the CSF; understanding the optical properties of each layer of the head, however, has allowed accurate modeling of fNIRS characteristics in the adult human brain (Hoshi, 2003).

In future studies, it may be worthwhile to compute neural synchronization between different regions of paired participants. Jiang et al. (2012) only compared synchrony between the same regions of each paired participant. Critically, however, the neural synchronization does not have to occur either at the same region or at the same time across the paired participants. Rather two brains may form a more complex dynamic system; for example, when two people interact, activity in the perceptual system of one brain is likely to be correlated, with some time delay, with activity in the motor system of the other brain (Hasson et al., 2012).

Face-to-face communication offers a superior form of communication in the context of this study. We communicate over the phone and by e-mail, but messages tend to get misinterpreted and a social connection can hardly be established or maintained. Jiang et al. (2012) found that face-to-face interaction increased the quality of communication as well as interbrain correlated activity, suggesting there is some literal truth to the expression "we are on the same wavelength". The study suggests that face-to-face communication has important neural and behavioral features that other types of communication cannot rival, and the interbrain correlation results may have implications for understanding the neural mechanisms of social interaction and diagnosing and de- veloping treatment of social deficits associated with autism.

\section{References}

Ahissar E, Nagarajan S, Ahissar M, Protopapas A, Mahncke H, Merzenich MM (2001) Speech comprehension is correlated with temporal response patterns recorded from auditory cortex. Proc Natl Acad Sci USA 98:13367-13372. CrossRef Medline

Arnal LH, Morillon B, Kell CA, Giraud AL (2009) Dual neural routing of visual facilitation in speech processing. J Neurosci 29:13445-13453. CrossRef Medline

Bunce SC, Izzetoglu M, Izzetoglu K, Onaral B, Pourrezaei K (2006) Functional near-infrared spectroscopy. IEEE Eng Med Biol Mag 25:54-62. CrossRef Medline

Chandrasekaran C, Trubanova A, Stillittano S, Caplier A, Ghazanfar AA (2009) The natural statistics of audiovisual speech. PLoS Comput Biol 5:e1000436. CrossRef Medline

Farrow TFD, Zheng Y, Wilkinson ID, Spence SA, Deakin JFW, Tarrier N, Griffiths PD, Woodruff PWR (2001) Investigating the functional anatomy of empathy and forgiveness. Neuroreport 12:2433-2438. CrossRef Medline

Hasson U, Ghazanfar AA, Galantucci B, Garrod S, Keysers C (2012) Brain-to-brain coupling: a mechanism for creating and sharing a social world. Trends Cogn Sci 16:114-121. CrossRef Medline

Hoshi Y (2003) Functional near-infrared optical imaging: utility and limitations in human brain mapping. Psychophysiology 40:511-520. CrossRef Medline

Jiang J, Dai B, Peng D, Zhu C, Liu L, Lu C (2012) Neural synchronization during face-to-face communication. J Neurosci 32:16064-16069. CrossRef Medline

Luo H, Poeppel D (2007) Phase patterns of neuronal responses reliably discriminate speech in human auditory cortex. Neuron 54:1001-1010. CrossRef Medline

Plichta MM, Gerdes AB, Alpers GW, Harnisch W, Brill S, Wieser MJ, Fallgatter AJ (2011) Auditory cortex activation is modulated by emotion: a functional near-infrared spectroscopy (fNIRS) study. Neuroimage 55:1200-1207. CrossRef Medline

Yun K, Watanabe K, Shimojo S (2012) Interpersonal body and neural synchronization as a marker of implicit social interaction. Sci Rep 2:959. Medline 